www.jmscr.igmpublication.org

Impact Factor (SJIF): 6.379

Index Copernicus Value: 79.54

ISSN (e)-2347-176x ISSN (p) 2455-0450

crossrefDOI: https://dx.doi.org/10.18535/jmscr/v6i9.155

Journal Of Medical Science And Clinical Research

IGM Publication

An Official Publication of IGM Publication

\title{
Hip Adductor Spasticity Scales and Walking Base of Support in Children with Cerebral Palsy
}

\author{
Authors \\ Abhimanyu Vasudeva ${ }^{1 *}$, Nonica Laisram², Asem Rangita Chanu ${ }^{3}$ \\ ${ }^{1}$ Senior Resident, Department of Physical Medicine and Rehabilitation, AIIMS, New Delhi, India \\ ${ }^{2}$ Consultant, Professor and Head, Department of Physical Medicine and Rehabilitation, Vardhman Mahavir \\ Medical College and Safdarjung Hospital, New Delhi, India \\ ${ }^{3}$ Assistant Professor, Department of Physical Medicine and Rehabilitation, AIIMS, New Delhi, India \\ *Corresponding Author
}

Abhimanyu Vasudeva

Address: A-1/124, Safdarjung Enclave, New Delhi, India 110029

Mobile: 759821688, Email: abhimanyu.vmmc@gmail.com

\begin{abstract}
Hip Adductor spasticity causes gait impairment in Cerebral Palsy (CP). Adductor Tone Rating Scale (ATRS) and Visual Method by vertical suspension are methods of grading this spasticity. The objective of our study was to study whether ATRS or Visual Method correlates better with walking Base of Support in CP children. It was an interventional cohort study in the outpatient clinic of Department of Physical Medicine and Rehabilitation in a Tertiary hospital. A total of 31 CP children with lower limb spasticity walking independently were enrolled. Botulinum toxin A was injected to gastrocnemius and hamstring with spasticity of Modified Ashworth Scale $\geq 2$ and/or Hip Adductors with ATRS $\geq 2$ and evaluated for various outcome measures before injection and at 4 and 12 weeks follow-up. Spearman's correlation analysis was performed between the walking base of support and each of ATRS and Visual Method. The main outcome measurements were ATRS and Visual Method for hip adductor spasticity, and walking base of support. Statistically significant reduction was seen after injection on ATRS and the Visual Method at 4 weeks and at 12 weeks compared with baseline. Significant improvement was seen in base of support of children with and without injection at 4 weeks ( $(p$ value $=0.011$ and 0.008$)$ and at 12 weeks ( $p$ value $=0.01$ land 0.007) compared with baseline. In the injected group, base of support had statistically significant correlation with Visual Method in contrast to ATRS at 4 weeks ( $p$ value $=0.0019$ and 0.0918) and at 12 weeks ( $p$ value $=0.0008$ and 0.6532). In non- injected group also, base of support had statistically significant correlation with Visual Method in contrast to ATRS at baseline and all follow - ups. To conclude, the Visual Method shows a better correlation than ATRS with walking base of support in CP children with lower limb spasticity.

Keywords: Cerebral Palsy, Spasticity, Gait, Botulinum toxin.
\end{abstract}

\section{Introduction}

Cerebral Palsy (CP) is a clinical syndrome characterized by a persistent disorder of posture or movement due to a non-progressive disorder of the immature brain. ${ }^{[1]}$ Spasticity is common in $\mathrm{CP}$ children and isolated Botulinum toxin A injections into the gastrocnemius muscle for equinus foot deformity, as well as multisite lower limb 
injections have shown improvements in gait and gross motor function. ${ }^{[2]}$ Hip adductor spasticity of the lower limbs often leads to decreased range-ofmotion (ROM), pain, disturbed gait and sitting position, as well as difficulties with perineal hygiene. ${ }^{[3]}$ The tools that are most frequently used for spasticity assessment in these children (the 'Ashworth- like scales') do not comply with the concept of spasticity, as it is velocity-dependent. Only the original Tardieu Scale remains a suitable instrument to measure spasticity. However, the original test seems very time consuming with lack of standardization of the muscle stretch velocities. ${ }^{[4]}$ A Visual Method by vertical suspension for grading hip adductor spasticity i.e., grade $1=$ touching at ankle, grade $2=$ crossing at ankle and grade $3=$ crossing at knee in spastic CP children with the child held in vertical suspension is gaining in popularity. ${ }^{[5]}$ The objective of this study was to assess which of the two scales of hip adductor spasticity measurement, Adductor Tone Rating Scale (ATRS) and Visual Method, correlates better with walking base of support in children with spastic cerebral palsy.

\section{Materials and Methods}

This study is a part of an interventional cohort study, conducted in the Out-Patient Department (OPD) of the Department of Physical Medicine and Rehabilitation of a Government Hospital in North India to evaluate the efficacy and functional outcome of Botulinum toxin type A in lower limb spasticity in cerebral palsy conducted from 26 12- 2013 to 25-12 -2014. After taking due approval from the Institute Ethics Committee, all $\mathrm{CP}$ children aged $\geq 2$ (two) years with lower limb spasticity of Modified Ashworth Scale (MAS) $\geq 2$ (two) for gastrocnemius and hamstrings and/or Adductor Tone Rating Scale $\geq 2$ (two) for Hip Adductor spasticity, and who can walk independently with or without support (stick/crutch/rollator) were enrolled in the study after taking informed consent from parents. However, those CP children with contracture or prior lower limb surgery, spasticity in the soleus muscle, taking antispastic medications, Botulinum toxin injection in the past 6 (six) months and unable to follow commands were excluded from the study. Reconstitution of each vacuum-dried vial (100 units) of Botulinum toxin A, available free of cost in our hospital supply, was done with $2 \mathrm{ml}$ of sterile $0.9 \%$ Sodium Chloride Injection which was administered within 24 hours of reconstitution. We used the lowest dose of Botulinum toxin A per unit body weight of the range suggested by Worldwide Education and Awareness for Movement Disorders ${ }^{[6]}$. Exercise program and splinting were continued. The Outcome Measures used were:

1. Adductor Tone Rating Scale (ATRS) for Hip Adductor spasticity ${ }^{[7]}$

2. Visual Method for Hip Adductor Spasticity $^{[5]}$

3. Base of Support as measured using the footprint analysis

Children were instructed to walk at their regular speed across a white paper looking straight ahead after having their feet dipped into a tray of tempera paint. The entire sequence was timed using a stopwatch. All the enrolled children were evaluated for the different outcome measures just before injection, at 4 and 12 weeks after injection (0, 4 and 12 weeks).Referring to the article by Raj et al. ${ }^{[8]}$, the minimum required sample size with $10 \%$ margin of error and $5 \%$ level of significance is 26 patients. To reduce the margin of error, total sample size was taken as 31 .

\section{Statistical Analysis}

All the categorical variables were presented in numbers and percentages (\%) while quantitative variables were presented as mean \pm standard deviation (median, range). Normality of data was tested by Kolmogorov-Smirnov test. Non parametric tests were done if the normality was rejected. Change in ATRS on follow-ups were analysed with Chi Square test. Change in Visual Method for Hip Adductor Spasticity at different follow-ups was analysed using Wilcoxon signedrank test. Spearman's correlation analysis was 
performed between the walking Base of Support and each of ATRS and Visual Method for Hip Adductor Spasticity on follow-ups. A p value of $<0.05$ was considered statistically significant. Analyses were done using SPSS version 21.0 Statistical Software (IBM Corp., Armonk, NY).

\section{Results}

CP children ranging from $4-14$ years of age were enrolled in the study. Out of them, 28 (90.3\%) children were in the age group of $4-9$ years and remaining $3(9.7 \%)$ children were above 9 years of age. Majority of them were males numbering $27(87.1 \%)$. All the enrolled children completed the study. Topographically, $21(67.7 \%)$ were diplegics, 3 (9.7\%) quadriplegics, 6 (19.4\%) right hemiplegics and $1(3.2 \%)$ left hemiplegic.

Botulinum toxin A was injected in all gastrocnemius and hamstring muscles with spasticity of grade $\geq 2$ (two) on Modified Ashworth Scale. It was also injected in Hip Adductor muscles that had spasticity grade $\geq 2$ (two) on the ATRS. There were 8 such CP children with Hip Adductor spasticity out of which 7 had bilateral Hip Adductor muscle injections. Statistically significant reduction was seen after local Botulinum toxin A injection in Hip Adductor muscle spasticity on ATRS and Visual Method at 4 weeks ( $\mathrm{p}$ value $=0.0004$ and 0.008 respectively) and at 12 weeks ( $\mathrm{p}$ value $=$ 0.0004 and 0.007 respectively) in these children compared with baseline but not at 12 weeks compared to 4 weeks ( $\mathrm{p}$ value $=1.000$ and 0.157 respectively) (Table-1).

Chi Square test for ATRS $(\mathrm{p}<0.05$ considered statistically significant) and Wilcoxon signed-rank test for Visual Method $(\mathrm{p}<0.05$ considered statistically significant) was applied.

Hip Adductor spasticity was also evaluated at different follow-ups for those CP children who didn't receive local Botulinum toxin A injection to Hip adductors. They were found to be statistically insignificant ( $\mathrm{p}$ value $>0.05)$ (Table-2).
Base of Support at different follow-ups for the 8 $\mathrm{CP}$ children receiving and remaining 23 children not receiving Botulinum toxin A injection to Hip Adductor muscles were analysed. Statistically significant improvement was seen in Base of Support of all these children in the follow-ups at 4 weeks $((\mathrm{p}$ value $=0.011$ and 0.008 respectively $)$ and at 12 weeks ( $\mathrm{p}$ value $=0.011$ and 0.007 respectively) compared with baseline but not at 12 weeks compared to 4 weeks.

In those $\mathrm{CP}$ children with Botulinum toxin $\mathrm{A}$ injection to Hip Adductors, there was a statistically insignificant correlation between the Visual Method grading and the walking Base of Support at baseline (0 weeks). But, there was a statistically significant strong negative correlation between the two at 4 weeks and 12 weeks (Table4).

A statistically significant strong negative correlation was found between the Visual Method scale and the walking Base of Support in children with Hip Adductors not injected with Botulinum toxin A at baseline and all follow - ups (Table-5).

Statistically insignificant correlation was found between ATRS and walking Base of Support in all children irrespective of receiving Botulinum toxin A injection to Hip Adductors. (Table-6,7).

We also did a correlation analysis between Visual Method and the Base of Support of all children irrespective of Botulinum toxin A injection at baseline (0 weeks), 4 weeks and 12 weeks. A statistically significant strong negative correlation was found at baseline and all follow-ups for all children (Table-8).

Correlation analyses between ATRS and the Base of Support of all Hip Adductors irrespective of Botulinum toxin A injection were also evaluated. A statistically significant strong negative correlation at baseline, and statistically significant moderate negative correlation at 4 and 12 weeks were found (Table-9). 
Table-1 ATRS and Visual Method grades of Hip Adductor spasticity at baseline and follow-ups for children receiving Botulinum toxin A injection to Hip Adductor muscle.

\begin{tabular}{|l|c|c|c|c|c|c|}
\hline & Mean \pm SD & Median & Min-Max & Inter quartile Range & \multicolumn{2}{|c|}{ P value } \\
\hline ATRS at 0 weeks & $2.4 \pm 0.51$ & 2 & $2-3$ & $2-3$ & \multicolumn{2}{|c|}{} \\
\hline ATRS at 4 weeks & $1 \pm 0.53$ & 1 & $0-2$ & $1-1$ & 0.0004 & \\
\hline ATRS at 12 weeks & $1 \pm 0.53$ & 1 & $0-2$ & $1-1$ & 0.0004 & 1 \\
\hline Visual Method at 0 weeks & $2.38 \pm 0.74$ & 2.5 & $1-3$ & $2-3$ & & \\
\hline Visual Method at 4 weeks & $1.5 \pm 0.93$ & 1.5 & $0-3$ & $1-2$ & 0.008 & \\
\cline { 1 - 6 } Visual Method at 12 weeks & $1.25 \pm 0.89$ & 1.5 & $0-2$ & $0.500-2$ & 0.007 & 0.157 \\
\hline
\end{tabular}

Table-2 ATRS and Visual Method grades of Hip Adductor spasticity at baseline and follow-ups for children not receiving Botulinum toxin A injection to Hip Adductor muscle. Chi Square test for ATRS ( $\mathrm{p}<0.05$ considered statistically significant) and Wilcoxon signed-rank test for Visual Method $(\mathrm{p}<0.05$ considered statistically significant) appliedweeks ( $\mathrm{p}$ value $=0.068$ and 0.948 respectively)

\begin{tabular}{|l|c|c|c|c|c|c|}
\hline & Mean \pm SD & Median & Min-Max & Inter quartile Range & \multicolumn{2}{|c|}{ P value } \\
\hline ATRS at 0 weeks & $0.28 \pm 0.45$ & 0 & $0-1$ & $0-1$ & \multicolumn{2}{|c|}{} \\
\hline ATRS at 4 weeks & $0.28 \pm 0.45$ & 0 & $0-1$ & $0-1$ & 1 & \\
\hline ATRS at 12 weeks & $0.28 \pm 0.45$ & 0 & $0-1$ & $0-1$ & 1 & \\
\hline Visual Method at 0 weeks & $0.22 \pm 0.67$ & 0 & $0-3$ & $0-0$ & & \\
\hline Visual Method at 4 weeks & $0.17 \pm 0.49$ & 0 & $0-2$ & $0-0$ & 0.317 & \\
\cline { 1 - 6 } Visual Method at 12 weeks & $0.13 \pm 0.34$ & 0 & $0-1$ & $0-0$ & 0.317 & 0.317 \\
\hline
\end{tabular}

Table-3.Walking Base of Support of all children at baseline and follow-ups. Chi Square test for Base of Support ( $\mathrm{p}<0.05$ considered statistically significant) applied

\begin{tabular}{|l|c|c|c|c|c|c|c|}
\hline $\begin{array}{l}\text { Base of Support } \\
\text { At }\end{array}$ & Sample size & Mean \pm SD & Median & Min-Max & $\begin{array}{c}\text { Inter quartile } \\
\text { Range }\end{array}$ & \multicolumn{2}{|c|}{ P value } \\
\hline 0 weeks & 8 & $-2.38 \pm 6.19$ & -4 & $-9-10$ & $-6.500-0.500$ & & \\
\hline 4 weeks & 8 & $3.12 \pm 5.38$ & 3 & $-3-13$ & $-1.500-6$ & 0.011 & \\
\cline { 1 - 7 } 12 weeks & 8 & $4.38 \pm 5.01$ & 3.5 & $-3-13$ & $1.500-7.500$ & 0.011 & 0.068 \\
\hline 0 weeks & 23 & $11.83 \pm 3.64$ & 12 & $-2-17$ & $11.250-14$ & & \\
\hline 4 weeks & 23 & $12.33 \pm 2.99$ & 12.5 & $2-17$ & $12-14.375$ & 0.008 & \\
\cline { 1 - 6 } 12 weeks & 23 & $12.61 \pm 2.17$ & 12.5 & $9-18$ & $12-14$ & 0.007 & 0.943 \\
\hline
\end{tabular}

Table-4.Spearman's correlation analysis between Visual Method grading and walking Base of Support in children receiving Botulinum toxin A injection to Hip Adductor muscle at baseline and follow-ups. Correlation is significant at the 0.05 level (2-tailed)

\begin{tabular}{|l|c|c|}
\hline $\begin{array}{l}\text { Visual Method and } \\
\text { Base of Support at } \\
(\mathrm{N}=8)\end{array}$ & Correlation Coefficient & P value \\
\hline 0 weeks & -0.548 & 0.1599 \\
\hline 4 weeks & -0.907 & 0.0019 \\
\hline 12 weeks & -0.931 & 0.0008 \\
\hline
\end{tabular}

Table-5 Spearman's correlation analysis between Visual Method grading and walking Base of Support in children not receiving Botulinum toxin A injection to Hip Adductor muscle at baseline and follow-ups. Correlation is significant at the 0.05 level (2-tailed)

\begin{tabular}{|l|c|c|}
\hline $\begin{array}{l}\text { Visual Method and } \\
\text { Base of Support at }(\mathrm{N}=23)\end{array}$ & $\begin{array}{l}\text { Correlation } \\
\text { Coefficient }\end{array}$ & $\mathrm{P}$ value \\
\hline 0 weeks & -0.576 & 0.004 \\
\hline 4 weeks & -0.581 & 0.0036 \\
\hline 12 weeks & -0.53 & 0.0093 \\
\hline
\end{tabular}


Table-6 Spearman's correlation analysis between ATRS and walking Base of Support in children receiving Botulinum toxin A injection to Hip Adductor muscle at baseline and follow-ups. Correlation is significant at the 0.05 level (2tailed)

\begin{tabular}{|l|c|c|}
\hline $\begin{array}{l}\text { ATRS and Base of } \\
\text { Support at } \\
(\mathrm{N}=15)\end{array}$ & Correlation Coefficient & $\begin{array}{c}\mathrm{P} \\
\text { value }\end{array}$ \\
\hline 0 weeks & -0.095 & 0.7361 \\
\hline 4 weeks & 0.485 & 0.0671 \\
\hline 12 weeks & 0.454 & 0.0889 \\
\hline
\end{tabular}

Table-7 Spearman's correlation analysis between ATRS and walking Base of Support in children not receiving Botulinum toxin A injection to Hip Adductor muscle at baseline and follow-ups. Correlation is significant at the 0.05 level (2tailed)

\begin{tabular}{|l|c|c|}
\hline $\begin{array}{l}\text { ATRS and Base of } \\
\text { Support at } \\
(\mathrm{N}=47)\end{array}$ & Correlation Coefficient & P value \\
\hline 0 weeks & 0.007 & 0.9621 \\
\hline 4 weeks & -0.004 & 0.9812 \\
\hline 12 weeks & 0.067 & 0.6532 \\
\hline
\end{tabular}

Table-8 Spearman's correlation analysis between Visual Method grading and walking Base of Support for all children at baseline and followups. Correlation is significant at the 0.05 level (2tailed)

\begin{tabular}{|l|c|c|}
\hline $\begin{array}{l}\text { Visual Method and } \\
\text { Base of Support at } \\
\text { (N=31) }\end{array}$ & Correlation Coefficient & P value \\
\hline 0 weeks & -0.825 & $<0.0001$ \\
\hline 4 weeks & -0.825 & $<0.0001$ \\
\hline 12 weeks & -0.764 & $<0.0001$ \\
\hline
\end{tabular}

Table-9 Spearman's correlation analysis between ATRS and walking Base of Support for all children at baseline and follow-ups. Correlation is significant at the 0.05 level (2-tailed)

\begin{tabular}{|l|c|c|}
\hline $\begin{array}{l}\text { ATRS and Base of } \\
\text { Support at } \\
(\mathrm{N}=62)\end{array}$ & Correlation Coefficient & $\mathrm{P}$ value \\
\hline 0 weeks & -0.592 & $<0.0001$ \\
\hline 4 weeks & -0.342 & 0.0065 \\
\hline 12 weeks & -0.303 & 0.0167 \\
\hline
\end{tabular}

\section{Discussion}

To move safely and efficiently from one place to another is the main functional goal of human ambulation. Normal ambulation depends on selective muscle control and normal range of motion of the joints. Defective motor control and limb deformities are common in upper motor neuron syndrome (UMNS). Spasticity is one of the components of UMNS. ${ }^{[9]}$ Hip Adductor muscle over activity is common in UMNS. Hygiene, dressing, sexual intimacy, sitting, transfers, standing, and walking can be adversely affected in patients with Hip Adductor spasticity. ${ }^{[10]}$ Hip Adductors over activity is characterized by adduction of the hip during the swing phase of gait, resulting in a narrow walking Base of Support in stance phase, with balance impairment. ${ }^{[11]}$ Gait analysis of patients with Hip Adductor spasticity has demonstrated a narrow Base of Support, poor standing balance, and unstable gait. ${ }^{[11,12,13]}$ Hip Adductor spasticity with scissoring thighs may also lead to impaired limb advancement and clearance.

Obturator neurolysis with a $7 \%$ phenol solution decreases Hip Adductor muscle over activity. ${ }^{[14]}$ Cerebral palsy (CP) is the most common neurologic disorder causing chronic disability in children and Hip Adductor spasticity has a great impact on developing hip displacement in these children. ${ }^{[15]}$ If left untreated, it may lead to dislocation, causing pain, gait disturbances, difficulty in sitting, and problems with perineal hygiene. ${ }^{[16]}$ In CP children with walking ability, open adductor tenotomy results in stabilisation or improvement in femoral-head subluxation. ${ }^{[17]}$ Recently, Botulinum toxin A injection to Hip Adductor muscles has been found to be beneficial in alleviating hip lateralization along with reducing Hip Adductor spasticity. ${ }^{[18,19,20]}$ Obturator neurolysis with phenol improves the walking Base of Support in subjects with Hip Adductor spasticity. ${ }^{[14]}$ Botulinum toxin A injection is found to be superior to phenol blocks in the reduction of spasticity, improvement in active range of motion and Gross Motor Function 
Measures in CP children with spastic diplegia. ${ }^{[21]}$ Botulinum toxin A injection also leads to more improvement in gait velocity and cadence in $\mathrm{CP}$ children compared to phenol block. ${ }^{[22]}$ As $\mathrm{CP}$ is dynamic in nature, continuous clinical assessment of child with treatment goals and planning is required. There is need of a dynamic tool to objectively quantify the motor functions of the $\mathrm{CP}$ child. This is provided by clinical gait analysis and guides the clinical treatment decision making in $\mathrm{CP}$ children. ${ }^{[23]}$

In our study, Botulinum toxin A resulted in improvement of Hip Adductor spasticity, measured by both ATRS and Visual Method. There was improvement in walking Base of Support in all CP children irrespective of Botulinum toxin A injection to Hip Adductor muscles. The continuation of exercises and splinting might be contributing factor to the improvement of walking Base of Support even in $\mathrm{CP}$ children not receiving the injection. The Visual Method of Hip Adductor muscle spasticity reflected in the improvement of walking Base of Support of all the CP children in this study. However, the ATRS was not reflective of the improvement in walking Base of Support even in those $\mathrm{CP}$ children receiving Botulinum toxin $\mathrm{A}$ injection.

This study is significant in the fact that the Visual Method by vertical suspension alongside ATRS might be a good approach in both assessment as well as treatment decision making in $\mathrm{CP}$ children with Hip Adductor spasticity as it correlates excellently with the walking Base of Support.

\section{Limitations}

It was a small sample size study with follow-ups only at 4 and 12 weeks after Botulinum toxin A injection. Only the Hip Adductor muscle spasticity was taken into consideration and not the cumulative effect of different muscles on gait. It focussed only on the walking Base of Support and not all parameters of gait.

\section{Conclusion}

To the best of our knowledge, this is the first study in India attempting to find if ATRS or the Visual Method by vertical suspension for Hip Adductor muscle spasticity is a better indicator of walking Base of Support in CP children. Local injection with Botulinum toxin A injection reduces the Hip Adductor spasticity in these children. The Visual Method shows a better indication of the improvement in walking Base of Support, if not other parameters of gait, than ATRS. Future studies of larger sample size with more frequent and longer duration of follow-ups and detailed gait analysis taking into consideration spasticity of different muscle groups is recommended.

\section{Acknowledgement}

I would like to acknowledge Mr Kulwant Singh Kapoor, Statistician, AIIMS, New Delhi for his guidance.

\section{Sources of support: None}

\section{References}

1. Mutch L, Alberman E, Hagberg B, Kodama K, Perat MV. (1992) Cerebral palsy epidemiology: where are we now and where are we going? Dev Med Child Neurol34: 547-551.

2. Olozek J, Davidson L. Cerebral Palsy. In: Braddom RL, editor. Physical medicine and rehabilitation. 4th edition. Philadelphia: Elsevier; 2011.p1235-55.

3. Wissel $\mathbf{J}^{1}$, Entner T. Wien Klin Wochenschr. 2001;113 Suppl 4:20 - 4 . Botulinum toxin treatment of hip adductor spasticity in multiple sclerosis. [Article in German]

4. Scholtes $\mathrm{VA}^{1}$, Becher JG, Beelen A, Lankhorst GJ. Clinical assessment of spasticity in children with cerebral palsy: a critical review of available instruments. Dev Med Child Neurol. 2006 Jan;48 (1):64-73. 
5. NonicaLaisram, Goyal Vinay. Grading of adductor spasticity in cerebral palsy - a new approach. IJPMR March 2015; Vol 26(1): 2-5.

6. WE MOVE. Management of Spasticity with Botulinum Toxin Type A (Botox), Edition 3.0. In, 2005.

7. Snow BJ, Tsui JK, Bhatt MH, Varelas M, Hashimoto SA, Calne DB. Treatment of spasticity with botulinum toxin: a doubleblind study. Ann Neurol. 1990;28(4):5125.

8. Raj K, Sanjay W, Singh U, Yadav SL. A study of effects of intervention of Botulinum toxin-A on lower limb in children with spastic cerebral palsy. IJPMR. 2015;26(4):94-101.

9. Mayer NH: Clinicophysiologic lesion concepts of spasticity and motor dysfunction in adults with an upper motoneuron lesion. Muscle Nerve 1997;20(suppl 6):S1 -13.

10. Mayer NH, Esquenazi A, Childers MK: Common patterns of clinical motor dysfunction. Muscle Nerve 1997;20(suppl 6): $\mathrm{S} 21-35$.

11. Esquenazi A: Computerized gait analysis for rehabilitation and surgical planning in upper motor neuron syndrome. Eur Med Phys 1999;35:111-8.

12. Mayer NH, Esquenazi A, Keenan MA: Analysis and management of spasticity contracture and impaired motor control after brain injury, in Horn LJ, Zasler ND (eds): Medical Rehabilitation of Traumatic Brain Injury. Philadelphia, Hanley and Belfus, 1996, pp 412-57.

13. Malanga G, DeLisa JA: Clinical observation, in DeLisa JA (ed): Gait Analysis in the Science of Rehabilitation. Baltimore, Baltimore Rehabilitation Information and Technology Administrative Center Department of Veterans Affairs, 1998, pp 1-11.
14. Ofluoglu D, Esquenazi A, Hirai B. Temporospatial parameters of gait after obturator neurolysis in patients with spasticity. Am J Phys Med Rehabil. 2003;82:832-836.

15. Eun Sook Park, Dong-WookRha, Won Chul Lee, and EunGeol Sim. The Effect of Obturator Nerve Block on Hip Lateralization in Low Functioning Children with Spastic Cerebral Palsy. Yonsei Med J 55(1):191-196, 2014.

16. Cooperman DR, Bartucci E, Dietrick E, Millar EA. Hip dislocation in spastic cerebral palsy: long-term consequences. J Pediatr Orthop 1987;7:268-76.

17. K. Pap, S. Kiss, T. Vízkelety, G. Szoke. Open adductor tenotomy in the prevention of hip subluxation in cerebral palsy. Int Orthop 2005; 29: 18-20.

18. Yang EJ, Rha DW, Kim HW, Park ES. Comparison of botulinum toxin type A injection and soft-tissue surgery to treat hip subluxation in children with cerebral palsy. Arch Phys Med Rehabil 2008;89:2108-13.

19. Jung NH, Heinen F, Westhoff B, Doederlein L, Reissig A, Berweck S, et al. Hip lateralisation in children with bilateral spastic cerebral palsy treated with botulinum toxin type A: a 2-year followup. Neuropediatrics 2011;42:18-23.

20. Graham HK, Boyd R, Carlin JB, Dobson F, Lowe K, Nattrass G, et al. Does botulinum toxin a combined with bracing prevent hip displacement in children with cerebral palsy and "hips at risk"? A randomized, controlled trial. J Bone Joint Surg Am 2008;90:23-33.

21. G Nitesh, L Vaibhav, A Mohammed, Gaur A, Shukla K. Phenol Versus Botulinum Toxin A Injection in Ambulatory Cerebral Palsy Spastic Diplegia: A Comparative Study. J PediatrNeurosc 2017; 12(4): 338343. 
22. Wong AM, Chen CL, Chen CP, Chou SW, Chung CY, Chen MJ. Clinical effects of botulinum toxin $\mathrm{A}$ and phenol block on gait in children with cerebral palsy. Am J Phys Med Rehabil 2004; 83(4):284-91.

23. Baker R, Esquenazi A, Benedetti MG, Desloovere K. Gait analysis: clinical facts. Eur J Phys Rehabil Med 2016;52:560-74. 